Research Article

\title{
Numerical investigation of the impact of inclined baffles and an elastic vibrating beam on the thermo-fluid behavior in a rectangular channel
}

\author{
S. Faramarzi ${ }^{1}$ (D) $\cdot$ R. GhasemiasI ${ }^{1} \cdot$ F. Ghadami ${ }^{2}$
}

Received: 6 July 2020 / Accepted: 23 March 2021

Published online: 23 April 2021

(c) The Author(s) 2021 OPEN

\begin{abstract}
In the present work, the influence of inclined baffles and an elastic vibrating beam is investigated on the flow pattern and heat transfer rate in a rectangular channel. The cylinder with the elastic vibrating beam develops the vibrating flow. The computations are based on the finite element method (FEM); Galerkin least-squares scheme and Newton-Raphson iterative method are implemented to solve the governing equations. The fluid structure-interaction (FSI) method is used to analyze the elastic vibrating beam. The friction factor coefficient and Nusselt number are examined for the inclined and conventional baffles. The flow regime is assumed to be laminar for various baffle angles and Reynolds numbers. The vibrating beam is connected to the cylinder located in the channel entrance. Optimized cases with the maximum heat transfer and minimum friction factor are introduced. The novelty of this study is the simultaneous use of the elastic vibrating beam and inclined baffles in a rectangular channel. The results show that mounting an elastic vibrating beam on the cylinder leads the heat transfer rate to increase. The channel with an elastic vibrating beam and inclined baffles at $135^{\circ}$ is the optimized case compared to other cases.
\end{abstract}

Article Highlights

- Take advantage of using an elastic vibrating beam in a rectangular channel.

- Influences of inclined baffles on thermal performance are examined numerically.
- The optimum case for the channel with inclined-baffles is reported.

Keywords Fluid-structure interaction $\cdot$ Navier-Stokes equations $\cdot$ Incompressible flow $\cdot$ Inclined baffle

\begin{tabular}{|c|c|c|c|}
\hline \multicolumn{2}{|c|}{ Abbreviations } & $f$ & Friction factor \\
\hline BR & The dimensionless ratio of baffle height to a chan- & $H$ & Channel height $(\mathrm{m})$ \\
\hline & nel $(b / H)$ & $L$ & Channel length $(\mathrm{m})$ \\
\hline 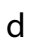 & Cylinder diameter (m) & $b$ & Baffle height (m \\
\hline$D$ & Relative Nusselt number dimensionless $\left(\frac{\overline{N u}_{\text {ave }}}{N(\mu)}\right)$ & $\mathrm{Nu}$ & Nusselt number \\
\hline$D_{\mathrm{h}}$ & $\begin{array}{l}\text { The hydraulic diameter of the rectangular channel } \\
(=2 \mathrm{H})\end{array}$ & $\begin{array}{l}p \\
\operatorname{Pr}\end{array}$ & $\begin{array}{l}\text { Pressure }(\mathrm{kPa}) \\
\text { Prandtl number }\end{array}$ \\
\hline$E$ & Thermal performance dimensionless $\left(\frac{\overline{N u}_{\text {ave }}}{N u_{0}} /\left(\frac{f}{f_{0}}\right)^{\frac{1}{3}}\right.$ & $\begin{array}{l}\operatorname{Re} \\
t\end{array}$ & $\begin{array}{l}\text { Reynolds number }\left(\rho \bar{u} D_{h} / \mu\right) \\
\text { Time (s) }\end{array}$ \\
\hline
\end{tabular}

S. Faramarzi, faramarzi.saman@wtiau.ac.ir | 'Department of Mechanical Engineering, West Tehran Branch, Islamic Azad University, Tehran, Iran. ${ }^{2}$ Department of Mechanical Engineering, Science And Research Branch, Islamic Azad University, Tehran, Iran. 
$T \quad$ Temperature $(\mathrm{k})$

$u \quad$ Velocity $\left(\mathrm{m} \mathrm{s}^{-1}\right)$

\section{Greek symbols}

$\theta \quad$ Prandtl number

$\mu \quad$ Dynamic viscosity

$\rho \quad$ Viscosity $\left(\mathrm{kg} \mathrm{m}^{-3}\right)$

\section{Subscripts}

ave The average upper and lower walls of the channel

$i, j \quad$ The vectors of the reference coordinates axis

o The channel without baffle and vibrating beam

w Channel wall

\section{Introduction}

Baffles are mounted in channels to alter the thermo-flow pattern. Vortices enhance the convection heat transfer by a significant change in streamlines. Many methods are used to improve heat transfer in a channel, including inclined baffles, porous baffles, and vibrating beam. Moreover, adding the baffles in channels causes a pressure drop. Optimized cases with maximum heat transfer rate and minimum pressure drop have been studied in many studies. Finding the optimized case is essential when the designer can not change its size and shape [1]. Inclined baffles are used in a 2D laser beam cutting machine[2].

$\mathrm{Li}$ et al. [3] investigated vibrating fluid flow around a smooth plane. They employed the control volume method and second-order scheme to solve the governing equations. Hu and Wang [4] introduced an approximate method to calculate the rubber plane's force. Promvonge et al. [5] investigated the effect of horse-shaped and inclined baffles experimentally. Wang et al. [6] investigated the effect of a porous baffle both experimentally and numerically on increasing heat transfer. The experimental results were obtained using the particle image velocimetry (PIV) method. Their numerical solution was based on a finite volume method. Wen et al. [7] investigated the effect of ladder-shaped multilayered baffles in heat exchangers both numerically and experimentally. The numerical research was based on a control volume solution and SIMPLE algorithm. The QUICK design was also employed with third-order accuracy for the discretization of the equations. Stelmach and Musoski [8] utilized PIV to investigate the flow inside a tank with four baffles. Promvonge et al. [9] investigated the effect of inclined rings on increasing heat transfer experimentally. The results indicated that those rings in the channel caused enhanced heat transfer and pressure drop.

Miranda et al. [10] studied the extent of forced convection heat transfer using the finite volume method in a two-dimensional channel with 16 baffles. The SIMPLEC algorithm was used. They also utilized porous baffles to minimize pressure drop in the channel. The effect of dimensionless quantities, including Reynolds number, Darcy number, heat transfer coefficient, and pressure drop, was also investigated. The Reynolds numbers were 100, 200,300 , and 400 . They found that the average Nusselt number of baffles with no holes was higher than that of the porous baffles. This difference was obtained for the Reynolds number 100, while by increasing the Reynolds number, the difference also improved. Porous baffles had a better thermal performance in comparison with normal ones. Mousavi and Hooman [11] studied forced convection heat transfer in a channel with baffles. The SIMPLER algorithm was used to link velocity and pressure equations. The linkage and discretization of diffusion and convection terms were performed by the power-law method. They examined the effect of Reynolds number, block ratio, and Prandtl number for different fluids in a laminar flow. They concluded that fluid with a larger Prandtl number caused improvement in Nusselt number value with the minimum pressure drop.

Siddiqui [12] experimentally and numerically studied the effect of forced convection heat transfer in a pipe with a circular section. The fluid velocity values were obtained using a digital PIV device. In the practical studies, a laser light source and a charge-coupled camera were employed. The numerical research was performed using the finite volume method and $k-\omega$ model. The SIMPLE algorithm was used to link the pressure and velocity equations. Discretization of the momentum equations was performed by the second-order upwind plan. The numerical and experimental results were compared and validated. They studied water fluid flow within the Reynolds range of $5000<\operatorname{Re}<20,000$ in a tube with a constant temperature wall. They concluded that increasing the vertical baffle height leads to a significant increase in Nusselt number and pressure drop.

On the other hand, the Nusselt number value was independent of the baffle angle for inclined baffles. They concluded that pressure drop increased with the increasing baffle angle. Overall, an increased Reynolds number caused an improvement in the Nusselt number.

Sripattanapipat and Promvonge [13] studied the properties of heat transfer and pressure drop in a channel with diamond-shaped baffles using the finite volume method. The SIMPLE algorithm was employed to solve velocity and pressure equations. Discretization of the governing equations was performed by QUICK design. The effect of the use of diamond-shaped baffles was examined in a channel with insulated walls. Reynolds numbers ranged between 100 and 600 . They concluded that using diamond-shaped baffles with an angle of $5^{\circ}$ increased the heat transfer coefficient by $6 \%$ compared to a flat baffle, whereas pressure 
drop did not change. The larger angle of diamond-shaped baffles decreased the heat transfer coefficient.

Promvonge et al. [14] investigated forced heat transfer in a baffled channel using the finite volume method. SIMPLE algorithm, QUICK discretization method, and a second-order upwind plan were used to solve the governing equations. The baffles were placed on the upper and lower walls of a channel along the same direction and angle. By analyzing the secondary flows, the effect of inclined baffles was examined on Nusselt number and friction factor. Reynolds numbers ranged between 100 and 1000. The dimensionless parameter of the channel thermal performance ratio $(E)$ was calculated for different states. Eventually, it was observed that inclined baffles had a better thermal performance compared to non-inclined baffles, where the optimal height of the baffle for the best thermal performance was 0.2

Pati et al. [15] studied forced convection heat transfer inside a wavy channel numerically. The wavelength of the walls influenced the dependence of the heat transfer on the geometry. Mehta and Pati [16] examined the entropy-generation characteristics and thermo-hydraulic performance of a triangular corrugated channel for Reynolds numbers 5-500. DeNayer et al. [17] investigated the classic problem associated with a flow behind a cylinder experimentally. They used an elastic plane behind the cylinder. Kalmbach and Breuer [18] investigated the developed vortexes experimentally using the FSI method. The experimental results were obtained using PIV. Turek and Hron [19] presented results associated with the FSI method related to incompressible flow around an elastic plane numerically. Sahin et al. [20] simulated and optimized the design parameters in a heat sink with trapezoidal baffles. The results showed that the Reynolds number was the most influential parameter on the heat transfer rate. The angle and the baffle width had a modest effect on the Nusselt number.

Mehta and Pati [21] did a numerical study for a wavy channel at different Prandtl numbers. Reynolds number, Prandtl number, and geometrical configuration of the channel strongly influenced the local thermal entropy generation distribution. Mehta and Pati [22] studied the thermal, hydraulic, and entropy generation characteristics of magneto-hydrodynamic pressure-driven flow of Al2O3water nanofluid in a wavy channel. They used Galerkin finite element method to solve the governing transport equations. The temperature and flow fields were investigated for different Reynolds numbers. Razavi et al. [23] studied incompressible laminar flow for a channel with and without baffles numerically. Inclined and perforated baffles were used in the channel to enhance the heat transfer rate.

In this study, simultaneous use of an elastic beam and inclined baffles are studied, which is the novelty of this work. Using a cylinder and elastic vibrating beam in a rectangular channel with inclined baffles has been examined. The FSI method based on the finite element method is implemented. The velocity and temperature fields are solved by the Galerkin scheme and the Newton-Raphson method. The impact of some parameter variations, including baffle angle, baffle height, and Reynolds number on the thermal performance ratio, the friction factor, and the flow pattern, is investigated. Given the results of this study, the channel with inclined baffles at $135^{\circ}$ and an elastic vibrating beam is the best case to enhance the heat transfer rate in a rectangular channel.

\section{Statement of the problem}

Two baffles have been placed in the channel, which has been investigated across different sizes and angles. Airflow is considered as the working fluid. The baffles have been examined in inclined and simple cases. Figure 1 demonstrates the overall schema of the channel.

Baffles are placed inside the channel. A solid cylinder and an elastic vibrating beam enhance the heat transfer rate (Fig. 1).

\subsection{The governing equations}

The Equations of continuity, momentum, and energy obtained for an incompressible fluid as follows:

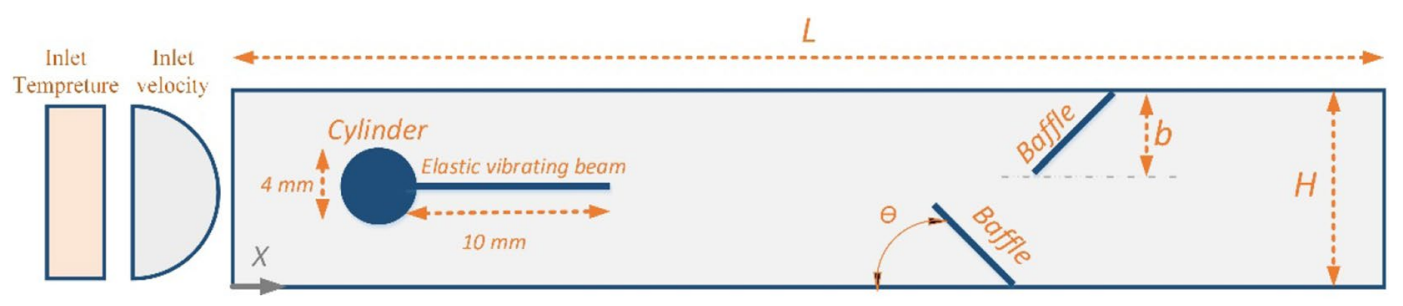

Fig. 1 Overall schema of the channel with an elastic vibrating beam and inclined baffles 
$\frac{\partial}{\partial x_{i}}\left(u_{i}\right)=0$

$$
\begin{aligned}
& \rho \frac{\partial}{\partial x_{j}}\left(u_{i} u_{j}\right)+\rho \frac{\partial}{\partial t}\left(u_{i}\right)=-\frac{\partial p}{\partial x_{i}} \\
& +\frac{\partial}{\partial x_{j}}\left[\mu\left(\frac{\partial u_{i}}{\partial x_{j}}+\frac{\partial u_{j}}{\partial x_{i}}\right)+\frac{\partial}{\partial x_{j}}\left[\mu\left(\frac{\partial u_{i}}{\partial x_{j}}+\frac{\partial u_{j}}{\partial x_{i}}\right)\right.\right. \\
& \rho c_{p} \frac{\partial}{\partial x_{j}}\left(u_{j} T\right)+\rho c_{p} \frac{\partial}{\partial t}(T)=\frac{\partial}{\partial x_{j}}\left(k \frac{\partial T}{\partial x_{j}}\right)
\end{aligned}
$$

FEM method is utilized to solve the governing Equations. Galerkin least-squares and Newton-Raphson iterative method have also been employed. A convergence analysis is also performed to find the minimum grid numbers (Fig. 2). Finally, 22,236 s-order elements and unstructured grids have been used to grid the model, as shown in Fig. 3 .

FSI method calculates quantities at the boundary between the fluid and structure in cases where the cylinder and vibrating beam are at the inlet of the channel. The EulerLagrangian method is utilized to combine the Equations associated with the fluid and structure. The force exerted to the middle layer and associated with the structure is calculated by Eq. 4 as follows:

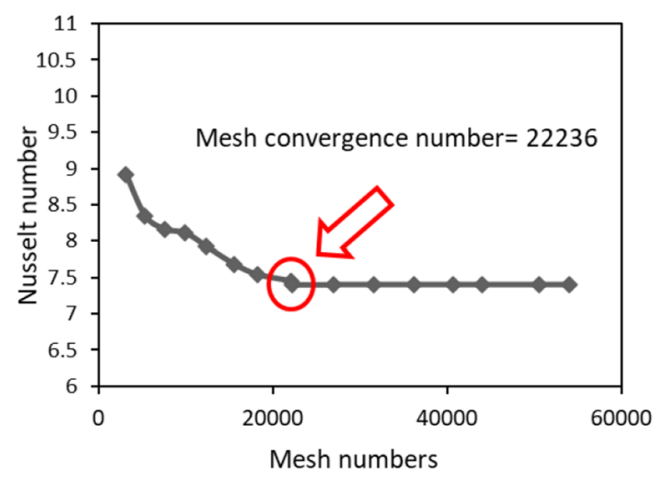

Fig. 2 Mesh convergence diagram, $\mathrm{Re}=200, \mathrm{~S}=0.2, \mathrm{BR}=0.4, \theta=90^{\circ}$
$F=n \cdot\left(-p l+\left(\mu\left(\nabla u_{f}+\left(\nabla u_{f}\right)^{T}-\frac{2}{3} \mu\left(\nabla \cdot u_{f}\right) l\right)\right)\right)$

where $F$ represents the force exerted to the fluid-structure boundary, $n$ denotes the normal vector on the boundary outwards, and $I$ is the unit matrix. In this paper, the FSI solution method is used based on the method presented by Turek and Hron [19] as well as Razzaq et al. [24].

For the cases where a cylinder and a vibrating beam have been used at the channel inlet, the fluid-structure interaction solution method is implemented to calculate quantities at the boundary between the fluid and structure. The Euler-Lagrangian method is utilized to combine the equations associated with the fluid and structure. The force exerted on the middle layer and associated with the structure is calculated as follows:

$F=n \cdot\left(-p l+\left(\mu\left(\nabla u_{f}+\left(\nabla u_{f}\right)^{T}-\frac{2}{3} \mu\left(\nabla \cdot u_{f}\right) l\right)\right)\right)$

where $F$ represents the force on the fluid-structure boundary, $n$ denotes the normal vector on the boundary outwards, and $I$ is the unit matrix.

\subsection{Initial and boundary conditions}

- The inlet of the channel has the conditions of parabolic velocity and constant temperature.

- The channel walls and baffles have non-slip and constant temperature conditions.

- The condition of the constant pressure of zero is considered in the outlet of the channel. In the cases where the cylinder and vibrating beam are used, the cylinder wall has the non-slip and thermal insulator conditions.

- The part of the vibrating beam connected to the cylinder has a fixed constraint.

- The channel's inlet temperature is $300 \mathrm{~K}$, while the temperature of the channel is $325 \mathrm{~K}$.

- The thickness and length of the elastic vibrating beam are $0.25 \mathrm{~mm}$ and $10 \mathrm{~mm}$, respectively. The diameter of the cylinder is $4 \mathrm{~mm}$.

- Reynolds number varies between 100 and 600 .

- The height of the channel $(\mathrm{H})$ is $10 \mathrm{~mm}$, and the baffle width is $0.02 \mathrm{H}$.

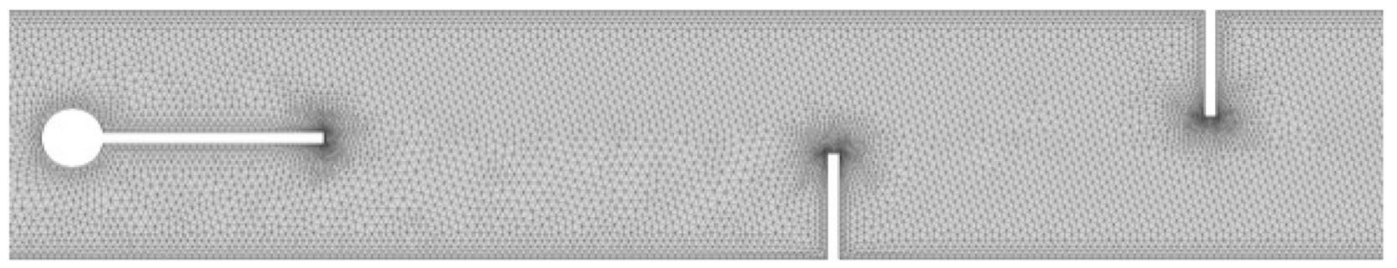

Fig. 3 Computational domain for the channel with a cylinder, an elastic beam, and two baffles $\left(S=0.2, B R=h / H=0.4, \theta=90^{\circ}\right)$ 


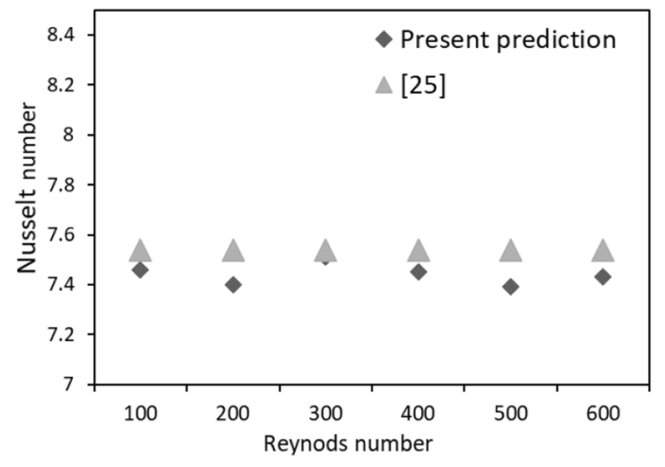

Fig. 4 Validation of the results associated with Nusselt number obtained for present work with that Bergman and Incropera [25]

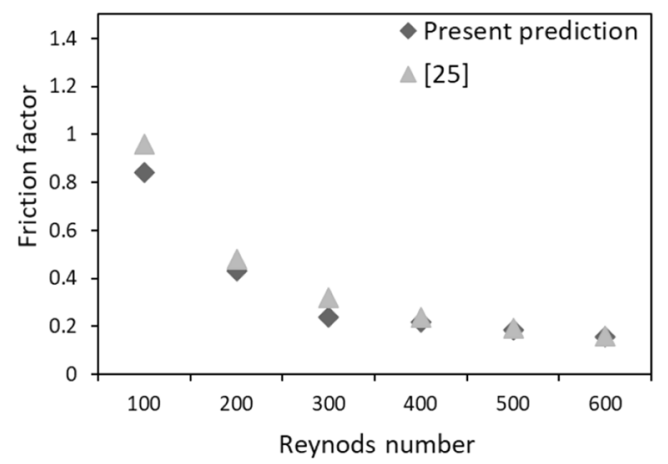

Fig. 5 Validation of the results related to friction coefficient obtained for present work with that Bergman and Incropera [25]

\section{Results and discussion}

Figures 4 and 5 validate the results associated with the Nusselt number and friction coefficient. The validation result is compared with Bergman and Incropera [25]. Accordingly, the results of the solution method have been confirmed.

\subsection{Inclined baffles}

Figures 6 and 7 demonstrate the effect of the dimensionless ratio of baffle height $(B R=h / H)$ on friction coefficient and Nusselt number. Based on these two figures, it is clear that with the increased $B R$, friction coefficient and relative Nussselt number increase. $f_{0}$ is the friction coefficient of a baffle-free channel, D represents the relative Nusselt number $\left(D=\frac{\overline{N u_{a v e}}}{N u_{0}}\right), N u_{\text {ave }}$ shows the average Nusselt number of the upper and lower walls, and $\mathrm{Nu}_{0}$ denotes the Nusselt number associated with the baffle-free channel.

The $45^{\circ}$ and $135^{\circ}$ baffles increased the area between the fluid and the wall compared to a $90^{\circ}$ baffle; therefore,

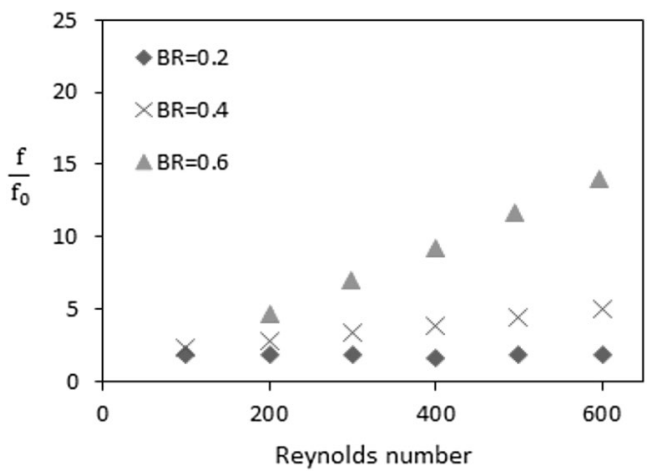

Fig. 6 The relative friction coefficient versus Reynolds number in the channel with simple baffle and different $B R s(B R=0.2,0.4$, and $\left.0.6, \theta=90^{\circ}\right)$

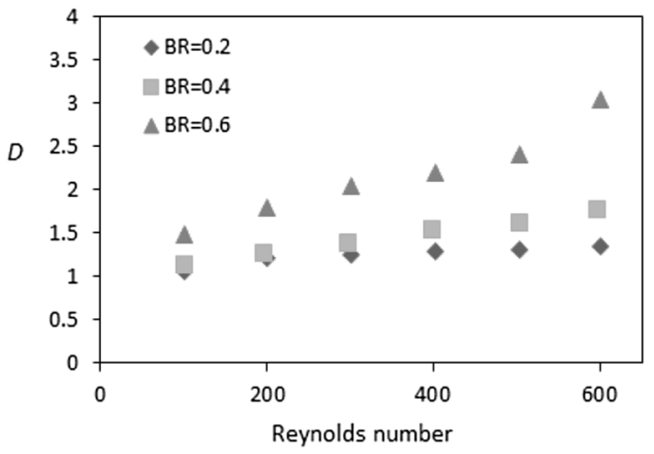

Fig. 7 Comparison of relative Nusselt number versus Reynolds number in the channel with simple baffle and different BRs $\left(\mathrm{BR}=\mathrm{h} / \mathrm{H}=0.2,0.4\right.$, and $\left.0.6, \theta=90^{\circ}\right)$

$45^{\circ}$ and $135^{\circ}$ baffles improve the heat transfer. At $B R=0.2$, increasing baffle angle has a slight effect on the Nusselt number. Due to the low height of the baffle, the vortexes that develop behind them are small, and a small area of the wall will be close to the vortex. Therefore, the temperature gradient increases in a smaller zone, and the Nusselt number will have less dependency on a low-height baffle. By increasing the low-height baffle angle, the size of vortexes undergoes a slight change, and thus the value of the relative Nusselt number changes very slightly. When the height ratio is $B R=0.4$ and 0.6 , increasing baffle angle causes a significant change in vortex power; thereby, it changes the local Nusselt number on the walls. Further, with increasing vortexes, the friction coefficient also changes.

The rotational flows behind the baffles grow by increasing Reynolds number. As a result, pressure drop and heat transfer increase; this is based on some definite reasons, and the most important of which is that the higher the velocity gradients, the more easily convection heat transfer occurs. Increasing the Reynolds number leads to more 
velocity gradient improvement along the channel. At $\mathrm{BR}=0.6$ and Reynolds number 600 , a leap occurs in friction coefficient and Nusselt number due to the beginning of the transition from laminar flow to turbulent flow. As the fluid velocity begins to fluctuate, momentum exchanges, and the heat transfer grows. When the dimensionless ratio of baffle height to a channel height $(B R=h / H)$ is maximum $(B R=0.6)$, the turbulence flow occurs more rapidly than baffles with a lower $B R(B R=0.2,0.4)$.

\subsection{Investigation of the thermal performance ratio}

The thermal performance ratio is defined to investigate the changes in Nusselt number and friction factor simultanously[6]:

$E=\frac{\frac{\overline{N u}_{\text {ave }}}{N u_{0}}}{\left(\frac{f}{f_{0}}\right)^{\frac{1}{3}}}$

Figures 8 and 9 reveal the thermal performance ratio for inclined baffles at different angles. The channel with an inclined baffle of $135^{\circ}$ and $B R=0.6$ has the best thermal performance than other baffles. The best thermal performance happens when the Nusselt number is maximum while the pressure drop is minimum. As Figs. 8 and 9, it is observed that inclined baffles have better performance in high Reynolds numbers.

Figure 8 presents that increasing Reynolds number leads to a significant change in thermal performance. At baffle angle $135^{\circ}$, a slight increase appears in Rey $=200$, continues with a sharp rise at Rey $=300$, reaching $E=1.14$. The thermal performance improves at $R e=500$, and it experiences a dramatic jump at Rey $=600$, peaking at $E=1.47$. Turning to the detail of.

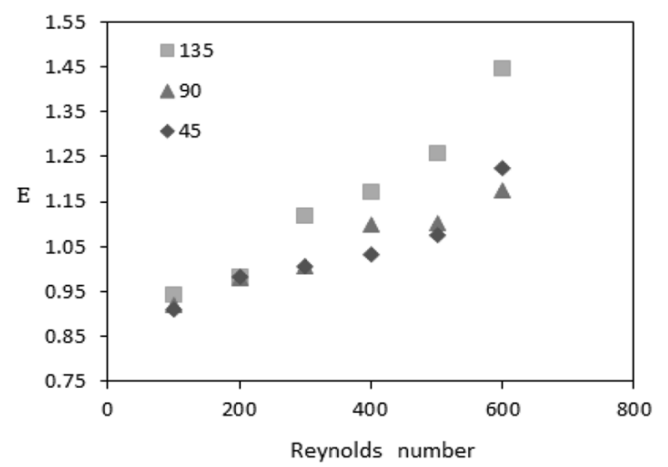

Fig. 8 Comparison of the thermal performance ratio versus Reynolds number in the channel with baffles and different $B R s(B R=0.6$, $\theta=45^{\circ}, 90^{\circ}$, and $135^{\circ}$ )

\section{SN Applied Sciences}

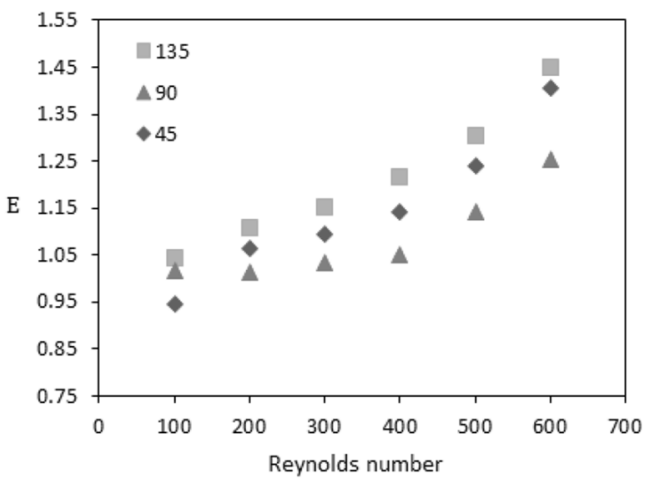

Fig. 9 Comparison of the thermal performance ratio versus Reynolds number in the channel with vibrating elastic beam and inclined baffles $\left(\mathrm{BR}=\mathrm{h} / \mathrm{H}=0.4, \theta=45^{\circ}, 90^{\circ}\right.$, and $\left.135^{\circ}\right)$

$\theta=90^{\circ}$, thermal efficiency improves from Rey $=100$ to Rey $=400$. It remains unchanged at $E=1.10$. The thermal efficiency of cases with $\theta=45^{\circ}$ increases in all Reynolds numbers, and it exceeds the cases of $90^{\circ}$.

It is clear from Fig. 8 that the case with a baffle angle of $135^{\circ}$ has the best performance among other cases.

Figure 9 has the same trend as Fig. 8. Although Fig. 9 has a smaller height than Fig. 8 , the best case is the same as in Fig. 8 , at $135^{\circ}$.

\subsection{Elastic vibrating beam}

Using an elastic vibrating beam behind a cylinder causes the development of unsteady flow. This unsteady flow behind the cylinder causes vibrating motion of the vibrating elastic beam attached to the cylinder, which in turn causes further fluctuation of flow downstream of the channel [26]. The vibration of the flow and using inclined baffles affect vortexes behind the baffles. Figure 10 shows the velocity field and streamlines downstream of the channel for five different time steps. Using the vibrating beam in this study increases the average heat transfer rate on the channel wall.

Figure 11 presents the effect of different baffle angles on streamlines in a channel at $\mathrm{Re}=600$ and $\mathrm{BR}=0.6$, in which two baffles are mounted vertically on the wall. Figure 11 a shows the streamlines for $\theta=135^{\circ}$, and Figs. $11 \mathrm{~b}$ and $11 \mathrm{c}$ show the streamlines for the angle of $45^{\circ}$ and $90^{\circ}$, respectively. Given the results of Figs. 8 and 9, the case with $\theta=135^{\circ}$ is the best case. The area between the flow and the wall in case $\theta=135^{\circ}$ is the largest compared to $\theta=45^{\circ}, 90^{\circ}$. It has the best heat transfer rate because the vortex located near the top baffle with $\theta=135^{\circ}$ is smaller than other cases. Shown in Fig. 12 is the comparison of cases with and without an elastic vibrating beam. 


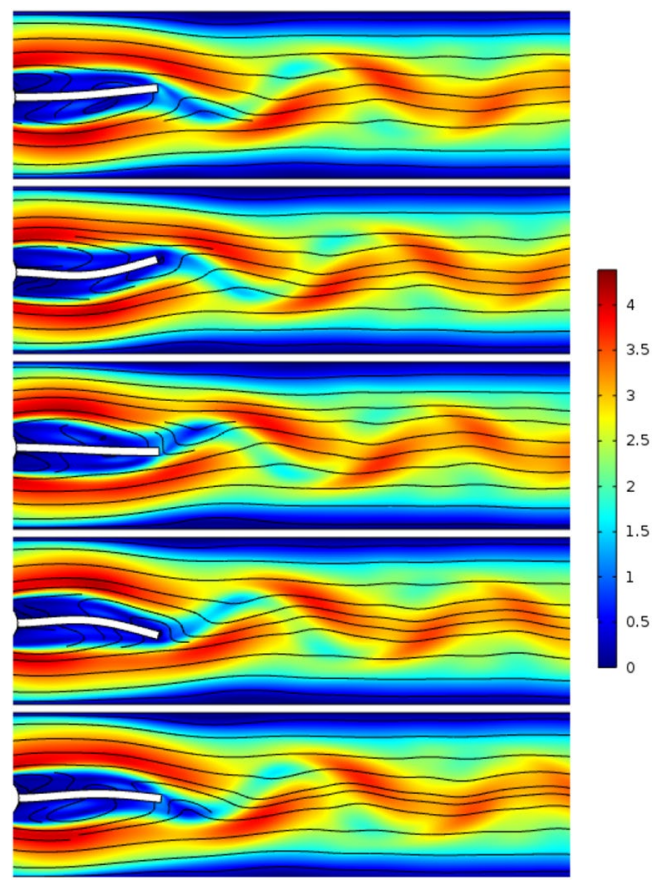

Fig. 10 Velocity field $(\mathrm{m} / \mathrm{s})$ and streamlines downstream of the channel for five different time steps for the best case

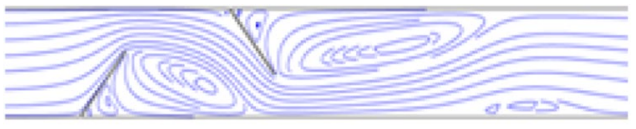

a

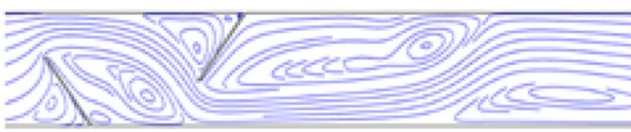

b

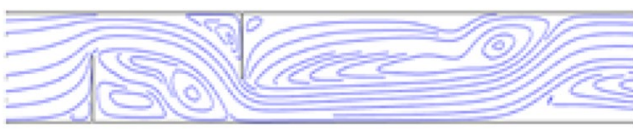

Fig. 11 Influence of inclination of baffles on the streamlines in three angles at $B R=0.6, \operatorname{Re}=600$ (Fig. $11 \mathrm{a}: \theta=135^{\circ}$, Fig. $11 \mathrm{~b}: \theta=45^{\circ}$, Fig. $11 \mathrm{C}=90^{\circ}$ )

Figure 12 shows the results of the channel with $135^{\circ}$ inclined baffles, a cylinder, and a vibrating beam. Using the vibrating beam causes instability in the flow; thus, the results have been reported as a mean value within a flow fluctuation period.

Based on Fig. 11, it is observed that the vibrating beam increases the heat transfer rate on the channel walls and also improves the average Nusselt number. Figure 13 shows the displacement of the tip of the elastic beam.

As shown in Fig. 13, the $y$-displacement of the tip of the elastic beam varies between $2 \mathrm{~mm}$ and $-2 \mathrm{~mm}$, while

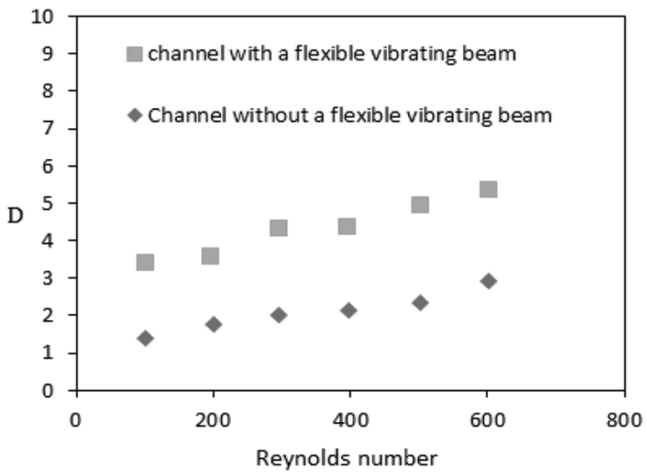

Fig. 12 Comparison of relative Nusselt number versus Reynolds number for the channel with and without elastic vibrating beam $\left(\mathrm{BR}=0.6, \theta=135^{\circ}\right)$

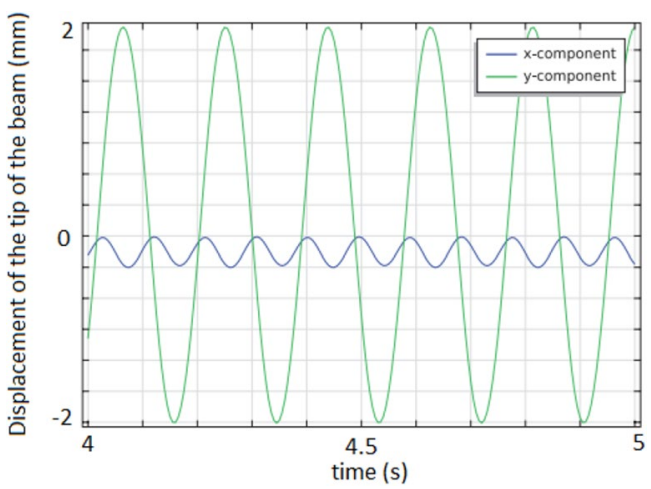

Fig. 13 Tip displacement of the elastic beam in the $\mathrm{x}$ and $\mathrm{y}$ directions

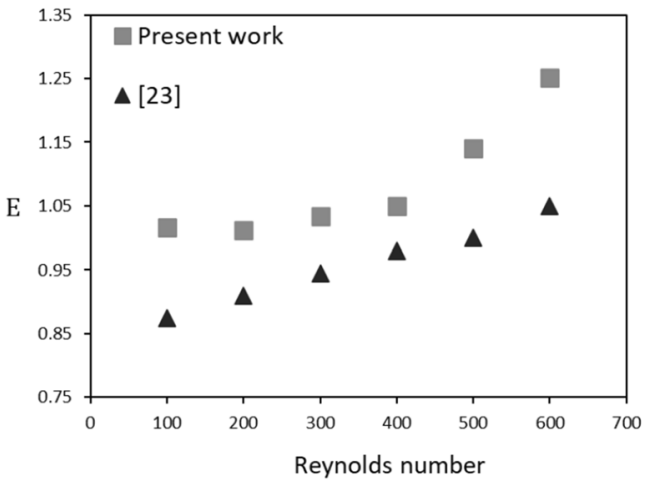

Fig. 14 Comparison of the thermal performance ratio between the present work and $[23]\left(\mathrm{BR}=\mathrm{h} / \mathrm{H}=0.4, \theta=90^{\circ}\right)$

the maximum is $2 \mathrm{~mm}$. Shown in Fig. 14 is the comparison between the model of the present work and the work of Razavi et al. [23]. It can be seen that the channel with a vibrating beam and baffles at $\theta=90^{\circ}$ performs better in terms of the thermal performance ratio than the model 
without a vibrating beam. Results from Fig. 14 indicate that using a vibrating beam can enhance the thermal efficiency of the channel for Reynolds numbers between 100 and 600 .

\section{Conclusion}

In this study, simultaneous use of an elastic vibrating beam and two inclined baffles are investigated numerically. Two-dimensional incompressible laminar flow in different angles, Reynolds numbers, and baffle heights is studied. An elastic vibrating beam causes primary vortices and increases the heat transfer rate. The thermal performance experiences a sharp rise when the height or the Reynolds number improves.

Using inclined baffles instead of conventional baffles causes better thermal performance only at $B R=0.6$. In some cases, inclined baffles decrease the thermal performance. Therefore The application of baffles in channels will have some limitations. The channel with inclined baffles at $135^{\circ}$ is the best case in which an elastic vibrating beam and a cylinder are at the inlet. At $E>1$, the thermal performance improves in comparison with the baffle-free channel. If $\mathrm{E}<1$, then using inclined baffles decreases the thermal performance. The relative Nusselt number has a direct relationship with the Reynolds number in all cases, which has an elastic vibrating beam.

Using a vibrating beam to enhance the heat transfer rate of a channel has limitations based on the location of the vibrating beam. The suitable location for the vibrating beam is near the inlet of the channel in which there are not any baffles. In future works, It is recommended to investigate the effect of using a vibrating beam for higher Reynolds numbers than 600 .

\section{Declarations}

Conflict of interest The authors declare that they have no conflict of interest.

Open Access This article is licensed under a Creative Commons Attribution 4.0 International License, which permits use, sharing, adaptation, distribution and reproduction in any medium or format, as long as you give appropriate credit to the original author(s) and the source, provide a link to the Creative Commons licence, and indicate if changes were made. The images or other third party material in this article are included in the article's Creative Commons licence, unless indicated otherwise in a credit line to the material. If material is not included in the article's Creative Commons licence and your intended use is not permitted by statutory regulation or exceeds the permitted use, you will need to obtain permission directly from the copyright holder. To view a copy of this licence, visit http://creativecommons. org/licenses/by/4.0/.

\section{References}

1. Jamalabadi MYA (2020) Optimal design of vibrating beam behind a cylinder. Ocean Eng 195:106759

2. Gu H, Chen Y, Wu J, Yang S (2017) Numerical study on performances of small incline angle helical baffle electric heaters with axial separation. Appl Therm Eng 126:963-975

3. Li G, Zheng Y, Yang H, Xu Y (2016) Numerical investigation of heat transfer and fluid flow around the rectangular flat plane confined by a cylinder under pulsating flow. J Appl Fluid Mech 9(4):1569-1577

4. Hu J, Wang W (2016) An Approximate Method for the Evaluation of the Normal Force Acting on a Flexible Plate Normal to the Wind Flow. J Appl Fluid Mech 9(4):1559-1568

5. Promvonge P, Tamna S, Pimsarn M, Thianpong C (2015) Thermal characterization in a circular tube fitted with inclined horseshoe baffles. Appl Therm Eng 75:1147-1155

6. Wang K, Tu X-C, Bae C-H, Kim H-B (2015) Optimal design of porous baffle to improve the flow distribution in the tube-side inlet of a shell and tube heat exchanger. Int J Heat Mass Transf 80:865-872

7. Wen J, Yang H, Wang S, Xue $Y$, Tong $X$ (2015) Experimental investigation on performance comparison for shell-and-tube heat exchangers with different baffles. Int J Heat Mass Transf 84:990-997

8. Stelmach J, Musoski R (2017) Hydrodynamics in the Blade Region of a Self-Aspirating Disk Impeller. J Appl Fluid Mech 10 (4):1177-1188

9. Promvonge $P$, Koolnapadol N, Pimsarn M, Thianpong C (2014) Thermal performance enhancement in a heat exchanger tube fitted with inclined vortex rings. Appl Therm Eng 62(1):285-292

10. Da Silva Miranda BM, Anand N (2004) Convective heat transfer in a channel with porous baffles. Numerical Heat Transfer Part A Appl 46(5):425-452

11. Mousavi S, Hooman K (2006) Heat and fluid flow in entrance region of a channel with staggered baffles. Energy Convers Manage 47(15-16):2011-2019

12. Siddiqui MK (2007) Heat transfer augmentation in a heat exchanger tube using a baffle. Int J Heat Fluid Flow 28(2):318-328

13. Sripattanapipat $S$, Promvonge $P$ (2009) Numerical analysis of laminar heat transfer in a channel with diamond-shaped baffles. Int Commun Heat Mass Transfer 36(1):32-38

14. Promvonge P, Sripattanapipat S, Kwankaomeng S (2010) Laminar periodic flow and heat transfer in square channel with 45 inline baffles on two opposite walls. Int J Therm Sci 49(6):963-975

15. Pati S, Mehta SK, Borah A (2017) Numerical investigation of thermo-hydraulic transport characteristics in wavy channels: comparison between raccoon and serpentine channels. Int Commun Heat Mass Transfer 88:171-176

16. Mehta SK, Pati S (2019) Analysis of thermo-hydraulic performance and entropy generation characteristics for laminar flow through triangular corrugated channel. J Therm Anal Calorim 136(1):49-62

17. De Nayer G, Kalmbach A, Breuer M, Sicklinger S, Wüchner R (2014) Flow past a cylinder with a flexible splitter plate: A complementary experimental-numerical investigation and a new FSI test case (FSI-PfS-1a). Comput Fluids 99:18-43

18. Kalmbach A, Breuer M (2013) Experimental PIV/V3V measurements of vortex-induced fluid-structure interaction in turbulent flow-a new benchmark FSI-PfS-2a. J Fluids Struct 42:369-387

19. Turek S, Hron J (2006) Proposal for numerical benchmarking of fluid-structure interaction between an elastic object and 
laminar incompressible flow. In: Bungartz HJ, Schäfer M (eds) Fluid-structure interaction. Springer, pp 371-385

20. Sahin B, Ates I, Manay E, Bayrakceken A, Celik C (2019) Optimization of design parameters for heat transfer and friction factor in a heat sink with hollow trapezoidal baffles. Appl Therm Eng 154:76-86

21. Mehta SK, Pati S (2020) Numerical study of thermo-hydraulic characteristics for forced convective flow through wavy channel at different Prandtl numbers. JThermal Anal Calorimet 141:1-23

22. Mehta SK, Pati S (2020) Thermo-hydraulic and entropy generation analysis for magnetohydrodynamic pressure driven flow of nanofluid through an asymmetric wavy channel. Int J Numer Methods Heat Fluid Flow 31(4):1190-1213

23. Razavi SE, Adibi T, Faramarzi S (2020) Impact of inclined and perforated baffles on the laminar thermo-flow behavior in rectangular channels. SN Appl Sci 2(2):284
24. Razzaq M, Turek S, Hron J, Acker J, Weichert F, Grunwald I, Roth C, Wagner M, Romeike_ B (2010) Numerical simulation and benchmarking of fluid-structure interaction with application to hemodynamics. In: Bungartz HJ, Mehl M, Schäfer M (eds) Fundamental Trends in Fluid-Structure Interaction. World Scientific, pp 171-199

25. Bergman TL, Incropera FP (2011) Fundamentals of heat and mass transfer. John Wiley \& Sons

26. Kundu A, Soti AK, Bhardwaj R, Thompson MC (2017) The response of an elastic splitter plate attached to a cylinder to laminar pulsatile flow. J Fluids Struct 68:423-443

Publisher's Note Springer Nature remains neutral with regard to jurisdictional claims in published maps and institutional affiliations. 